\title{
Geometrically Nonlinear Analyses Using 2-Dimensional Generalized Finite Element Enriched by the Quadratic Deformation Mode*
}

\author{
Yuichi TADANO $^{* *}$ and Hirohisa NOGUCHI ${ }^{* * *}$ \\ ** Department of Mechanical Engineering, Saga University, \\ 1 Honjo-machi, Saga 840-8502, Japan \\ E-mail: tadano@me.saga-u.ac.jp \\ *** Department of System Design Engineering, Keio University, \\ 3-14-1 Hiyoshi, Kouhoku-ku, Yokohama 223-8522, Japan \\ (Passed away on August 22, 2008)
}

\begin{abstract}
A formulation of a novel in-plane generalized finite element, which can be used in geometrically nonlinear analysis, is presented and some analyses are conducted using the proposed element. The presented element has four nodes, and four additional degrees of freedom are added on each node. As a result, this element can reproduce the quadratic deformation mode with only corner nodes and has no linear dependency, which is a well known problem of generalized finite elements. The formulation is based on the rate form of the virtual work principle and is obtained by a simple extension of the standard FEM. The convergence of the analysis solution and its robustness for element distortion are investigated, and the results are compared with those of standard-displacement-based first- and second-order elements. The proposed element provides solution convergence as good as or better than those of the conventional second-order elements. In addition, it is shown that an accurate solution is obtained when the mesh is strongly distorted.
\end{abstract}

Key words: Generalized Finite Element Method, Geometrically Nonlinear Analysis, Linear Independency, Compatible Element, Locking Free

\section{Introduction}

To improve the accuracy of the finite element method (FEM), many approaches have been proposed; one of the most practical and best-known ways is the generalized finite element method (GFEM). GFEM was introduced by several researchers in the $1980 \mathrm{~s}^{(1),(2)}$. The main concept of GFEM is that the precision of a solution can be refined drastically by the introduction of additional nodal degrees of freedom. In the recent decade, an enhancement of GFEM based on the partition of unity method (PUM), which was first introduced by Babuška ${ }^{(3)}$, has been actively pursued ${ }^{(4)-(8)}$. The idea of the partition of unity approach has good compatibility with the GFEM framework, although it was originally used in a type of mesh-free method $^{(9)}$. GFEM with PUM can improve the accuracy of FEM drastically by a simple extension of the conventional finite element formulation. It is also known that the conventional FEM is a special case in the GFEM framework ${ }^{(10)}$.

This approach, however, has several numerical shortcomings, such as the computational cost is high due to the increase in the degrees of freedom and the stiffness matrix becomes singular because of the linear dependence problem. As another approach to increase the performance of FEM, a finite element with drilling freedom was first proposed by 
Allman ${ }^{(11)}$. This is an extension of a triangular element and many modifications have been subsequently presented ${ }^{(12),(13)}$. In Allman's element, each element node has an independent additional degree of freedom that describes in-plane rotation. This enhances the solution accuracy for several problems, especially those with bending deformation. Recently, Sekiguchi and Kikuchi reviewed and re-examined the elements with drilling freedom and proposed a new element formulation, including a drilling freedom in the framework of the standard displacement method ${ }^{(14)}$. This element can be interpreted as an element based on the GFEM framework.

Despite the superior performance of GFEM, most studies focus only on linear problems and few nonlinear analyses have been reported. Barros et al. introduced GFEM into material nonlinear analysis ${ }^{(15)}$, and Terada et al. analyzed discontinuous surfaces and debonding problems by means of the finite cover method, which is a type of $\mathrm{GFEM}^{(16)}$. However, geometrical nonlinearity in GFEM has not been studied.

In this study, a novel in-plane generalized finite element enriched by the quadratic deformation mode is proposed in the framework of GFEM and geometrically nonlinear analyses are conducted. The presented element has only four corner nodes, and four additional degrees of freedom are added on each node. As a result, this element can reproduce the quadratic deformation mode with only corner nodes. The convergence of the analysis solution and the robustness for element distortion are investigated through comparison with the standard displacement based first and second-order elements.

\section{Formulation}

\subsection{Governing equations}

In this study, the formulation is based on the rate form of the equilibrium equation. Therefore, the governing equation is the rate form of Cauchy's first law of motion:

$$
\nabla \cdot \dot{\boldsymbol{\pi}}+\rho \dot{\mathbf{g}}=\mathbf{0}
$$

In this equation, the inertial force is neglected because only a static condition is considered. $\pi, \rho$, and $\mathbf{g}$ are the first Piola-Kirchhoff stress tensor, density, and body force vector, respectively. They are functions of the coordinates. $\nabla$ is the gradient operator and the superposed dot denotes the material time derivative.

The rate form of the virtual work principle based on Eq. (1), in which volumetric force is neglected, is given as

$$
\int_{V} \dot{\boldsymbol{\pi}}: \delta \mathbf{L}=\int_{S} \dot{\mathbf{t}} \cdot \delta \dot{\mathbf{u}} \mathrm{d} s
$$

where $\mathbf{L}, \mathbf{t}$, and $\mathbf{u}$ are the velocity gradient tensor, traction vector, and displacement vector, respectively. $\delta$ indicates the virtual quantities, and $v$ and $s$ are the current volume and surface, respectively. For the constitutive equation, material objectivity should be considered because finite rotation occurs in geometrically nonlinear analysis. Therefore, in the present formulation, the following constitutive relationship between the deformation rate tensor (i.e., the symmetric part of velocity gradient) $\mathbf{D}$ and the Jaumann rate of the Cauchy stress is assumed:

$$
\begin{aligned}
& \stackrel{\circ}{\boldsymbol{\sigma}}=\mathbf{C}: \mathbf{D} \\
& \stackrel{\circ}{\boldsymbol{\sigma}} \equiv \dot{\boldsymbol{\sigma}}-\mathbf{W} \boldsymbol{\sigma}+\boldsymbol{\sigma} \mathbf{W}
\end{aligned}
$$

$\dot{\sigma}$ denotes the Jaumann derivative of Cauchy stress, $\mathbf{C}$ is the fourth-order constitutive tensor, and $\mathbf{W}$ is the asymmetric part of the velocity gradient tensor. The relationship 
between the first Piola-Kirchhoff stress rate and the Cauchy stress rate is expressed as

$$
\dot{\boldsymbol{\pi}}=\dot{\boldsymbol{\sigma}}-\mathbf{L} \boldsymbol{\sigma}+(\operatorname{tr} \mathbf{L}) \boldsymbol{\sigma}
$$

Substituting Eqs. (3) and (4) into Eq. (5) yields

$$
\dot{\boldsymbol{\pi}}=\mathbf{C}: \mathbf{D}-\mathbf{D} \boldsymbol{\sigma}-\boldsymbol{\sigma} \mathbf{D}+\boldsymbol{\sigma} \mathbf{L}^{\mathrm{T}}+(\operatorname{tr} \mathbf{L}) \boldsymbol{\sigma}
$$

where the relation $\mathbf{W} \boldsymbol{\sigma}-\boldsymbol{\sigma} \mathbf{W}-\mathbf{L} \boldsymbol{\sigma}=-\mathbf{D} \boldsymbol{\sigma}-\boldsymbol{\sigma} \mathbf{D}+\boldsymbol{\sigma} \mathbf{L}^{\mathrm{T}}$ is used. By substituting Eq. (6) into Eq. (2) and separating terms with respect to the deformation rate and velocity gradient, the following relation can be obtained as the governing equation.

$$
\int_{v}\left[(\mathbf{C}: \mathbf{D}-\mathbf{D} \boldsymbol{\sigma}-\boldsymbol{\sigma D}): \delta \mathbf{D}+\left\{\boldsymbol{\sigma} \mathbf{L}^{\mathrm{T}}+(\operatorname{tr} \mathbf{L}) \boldsymbol{\sigma}\right\}: \delta \mathbf{L}\right] \mathrm{d} v=\int_{s} \dot{\mathbf{t}} \cdot \delta \dot{\mathbf{u}} \mathrm{d} s
$$

\subsection{Interpolation function}

The general expression of an interpolated displacement field $u$ in the GFEM framework is given as

$$
u=\sum_{n=1}^{m}\left[\sum_{j} L_{j} a_{j}^{n}\right] N^{n}
$$

Here, $L_{j}$ and $N^{n}$ are polynomial terms and the shape function, respectively. $a_{j}^{n}$ represents unknown coefficients. $m$ is the number of nodes in the element and the superscript $n$ denotes the values at the $n$-th node. Equation (8) is reduced to the conventional FEM formulation when $L_{j}=\{1\}$. In the nonlinear analysis based on the rate form equation, Eq. (8) should be applied to interpolate the velocity field instead of the displacement field. Therefore, the displacement terms in the equation are replaced by the displacement rates $\dot{u}$,

$$
\dot{u}=\sum_{n=1}^{m}\left[\sum_{j} L_{j} a_{j}^{n}\right] N^{n}
$$

Wilson proposed a 4-node element using the following shape function ${ }^{(17),(18)}$.

$$
u=\sum_{n=1}^{4} N^{n} u^{n}+\left(1-\xi^{2}\right) a^{n}+\left(1-\eta^{2}\right) b^{n}
$$

Here $N^{n}$ is the bilinear shape function identical to that in the conventional in-plane first-order element, $\xi$ and $\eta$ are coordinates in the natural coordinate system of the element, and $\alpha^{n}$ and $b^{n}$ are additional degrees of freedom on node $n$. This interpolation function can reproduce the quadratic deformation mode, which means that the displacement field expressed by the quadratic polynomial equation on coordinates can be described exactly. Equation (10) provides a discontinuous displacement field between elements; therefore, this element is a nonconforming element.

Motivated by Wilson's formulation, in this paper, the 4-node conventional linear element is enriched by the reduced quadratic polynomial terms:

$$
L_{j}=\left\{1,\left(x_{1}\right)^{2},\left(x_{2}\right)^{2}\right\}
$$




$$
\begin{aligned}
& 1 \\
& \begin{array}{ll}
x_{1} & x_{2}
\end{array} \\
& \left(x_{1}\right)^{2} \quad x_{1} x_{2} \quad\left(x_{1}\right)^{2} \\
& \left(x_{1}\right)^{3} \quad\left(x_{1}\right)^{2} x_{2} \quad x_{1}\left(x_{2}\right)^{2} \quad\left(x_{2}\right)^{3} \\
& \left(x_{1}\right)^{3} x_{2} \quad x_{1}\left(x_{2}\right)^{3}
\end{aligned}
$$

Fig. 1 Polynomial terms in the present formulation.

It is noted that the coordinates $x_{1}$ and $x_{2}$ are defined in the global coordinate system, although those of Wilson's element are in the natural coordinate system. Using Eq. (11) in the framework of GFEM (8), the interpolation functions are written as

$$
\begin{aligned}
& \dot{u}_{1}=\sum_{n=1}^{4}\left[\dot{u}_{1}^{n}+\left\{\left(x_{1}\right)^{2}-\left(x_{1}^{n}\right)^{2}\right\} \dot{a}^{n}+\left\{\left(x_{2}\right)^{2}-\left(x_{2}^{n}\right)^{2}\right\} \dot{b}^{n}\right] N^{n} \\
& \dot{u}_{2}=\sum_{n=1}^{4}\left[\dot{u}_{2}^{n}+\left\{\left(x_{1}\right)^{2}-\left(x_{1}^{n}\right)^{2}\right\} \dot{c}^{n}+\left\{\left(x_{2}\right)^{2}-\left(x_{2}^{n}\right)^{2}\right\} \dot{d}^{n}\right] N^{n}
\end{aligned}
$$

Here, $\dot{u}_{1}^{n}$ and $\dot{u}_{2}^{n}$ are the nodal displacements, and $\dot{a}^{n}, \dot{b}^{n}, \dot{c}^{n}$, and $\dot{d}^{n}$ are additional degrees of freedom. $N^{n}$ is the bilinear shape function of the conventional in-plane first-order element. This formulation can describe the quadratic deformation mode with only the corner nodes as same as Wilson's element. All terms, which can be expressed by the present element with a nondistorted rectangular shape, are shown in Fig. 1, which includes a complete perfect cubic polynomial terms; therefore the cubic displacement filed can be reproduced with a nondistorted finite element mesh. When an element is distorted, several terms in Fig. 1 are spoiled; however, the quadratic terms of $x_{1}$ and $x_{2}$ always remains. As a result, this element certainly produces the quadratic deformation mode. The displacement field is obtained by integrating Eqs. (12) and (13), and it cannot be expressed explicitly.

An important advantage of the present element over Wilson's one is that Eqs. (12) and (13) provide a compatible displacement field, although the original formulation (10) is a nonconforming or incompatible element. Therefore, this element satisfies the compatibility condition between elements. In addition, the formulation is based on the GFEM framework (8), the partition of unity condition is satisfied, and it is guaranteed that the constant strain field can be reproduced. These properties provide that the solution is converged with respect to the number of elements. It is a significant feature of the present formulation.

The linear dependency is a well-known problem of GFEM, which causes a lack of uniqueness of solution, because of duplicated polynomial terms. However, no linear dependency problem occurs with the present element, because Eqs. (12) and (13) can represent a displacement rate field without any duplicated terms. It is another important aspect of this element.

\subsection{Finite element discretization}

The discretization form of the left-hand side of Eq. (7), which corresponds to the internal force, is given as

$$
\{\delta \underline{\dot{\mathbf{U}}}\}\{\underline{\dot{\mathbf{Q}}}\}=\{\delta \underline{\mathbf{U}}\}[\underline{\mathbf{K}}]\{\underline{\dot{\mathbf{U}}}\}
$$

$\{\delta \underline{\mathbf{U}}\},\{\underline{\mathbf{U}}\}$, and $\{\underline{\mathbf{Q}}\}$ are the discretized virtual displacement rate, the displacement rate, and the internal force rate, respectively, and $[\underline{\mathbf{K}}]$ is the tangent stiffness matrix. A matrix or vector with an underbar is a quantity corresponding to the total system equation. Due to the arbitrary property of the virtual displacement rate, the following relation is obtained in 
each element:

$$
\begin{aligned}
\{\dot{\mathbf{Q}}\} & =[\mathbf{K}]\{\dot{\mathbf{U}}\} \\
\{\dot{\mathbf{U}}\} & \equiv\left\{\begin{array}{llll}
\dot{\mathbf{U}}^{1} & \dot{\mathbf{U}}^{2} & \dot{\mathbf{U}}^{3} & \dot{\mathbf{U}}^{4}
\end{array}\right\}
\end{aligned}
$$

The discretized external force $\{\dot{\mathbf{F}}\}$ of an element is

$$
\{\dot{\mathbf{F}}\} \equiv\left\{\begin{array}{llll}
\dot{\mathbf{F}}^{1} & \dot{\mathbf{F}}^{2} & \dot{\mathbf{F}}^{3} & \dot{\mathbf{F}}^{4}
\end{array}\right\}
$$

The tangent stiffness matrix $[\mathbf{K}]$ consists of terms corresponding to deformation rate $\mathbf{D}$ and velocity gradient $\mathbf{L}$. Therefore, it can be rewritten as

$$
\begin{aligned}
& {[\mathbf{K}]=\left[\mathbf{K}_{\mathrm{D}}\right]+\left[\mathbf{K}_{\mathrm{L}}\right]} \\
& {\left[\mathbf{K}_{\mathrm{D}}\right] \equiv \int_{v^{\mathrm{e}}}\left[\mathbf{B}_{\mathrm{D}}\right]^{\mathrm{T}}\left[\mathbf{D}_{\mathrm{D}}\right]\left[\mathbf{B}_{\mathrm{D}}\right] \mathrm{d} v^{\mathrm{e}}} \\
& {\left[\mathbf{K}_{\mathrm{L}}\right] \equiv \int_{v^{\mathrm{e}}}\left[\mathbf{B}_{\mathrm{L}}\right]^{\mathrm{T}}\left[\mathbf{D}_{\mathrm{L}}\right]\left[\mathbf{B}_{\mathrm{L}}\right] \mathrm{d} v^{\mathrm{e}}}
\end{aligned}
$$

where $v^{\mathrm{e}}$ is the volume of the element. $\left[\mathbf{B}_{\mathrm{D}}\right]$ and $\left[\mathbf{B}_{\mathrm{L}}\right]$ are matrices that translate the displacement rate vector into the deformation rate and the velocity gradient. Each component can be obtained easily in the same manner as that of the general nonlinear finite element formulation based on the updated Lagrangian method. $\dot{\mathbf{U}}^{n}$ in Eq. (16) is a vector that includes unknown coefficients at each node and is written as

$$
\left\{\dot{\mathbf{U}}^{n}\right\} \equiv\left\{\begin{array}{llllll}
\dot{u}_{1}^{n} & \dot{u}_{2}^{n} & \dot{a}^{n} & \dot{b}^{n} & \dot{c}^{n} & \dot{d}^{n}
\end{array}\right\}
$$

To prescribe the displacement of a node, $\dot{u}_{1}^{n}$ and/or $\dot{u}_{2}^{n}$ must be fixed the same as the general finite element method, and the additional degrees of freedom must be free. When the displacement must be prescribed along the element edge, constraints on the additional degrees of freedom are also needed. Namely, $\dot{a}^{n}=0$ and $\dot{b}^{n}=0$ when $\dot{u}_{1}^{n}$ is prescribed, and $\dot{c}^{n}=0$ and $\dot{d}^{n}=0$ when $\dot{u}_{2}^{n}$ is prescribed. The present element can avoid the difficulty of prescribing boundary conditions that may arise in some kind of mesh-free or generalized finite element method.

$\dot{\mathbf{F}}^{n}$ in Eq. (17) is an external force rate vector whose components correspond to those in $\dot{\mathbf{U}}^{n}$. Discretizing the virtual work by surface loading yields the concrete form of $\dot{\mathbf{F}}^{n}$. The virtual work caused by surface loading on an element surface $\delta \dot{W}^{\text {ext }}$ is equivalent to the right-hand side of Eq. (2).

$$
\begin{aligned}
\delta \dot{W}^{\text {ext }} & =\{\delta \dot{\mathbf{U}}\}\{\dot{\mathbf{F}}\} \\
& =\int_{s^{\mathrm{e}}} \dot{\mathbf{t}} \delta \dot{\mathbf{u}} \mathrm{d} s \\
& =\int_{s^{e}}\left(\dot{t}_{1} \delta \dot{u}_{1}+\dot{t}_{2} \delta \dot{u}_{2}\right) \mathrm{d} s
\end{aligned}
$$

where $s^{\mathrm{e}}$ is the area of the boundary surface. Substituting Eqs. (12) and (13) into the above equation yields the components of $\dot{\mathbf{F}}^{n}$. If a concentrated load is subjected on a node, it can be treated as a nodal force the same as the conventional element. On the other hand, note that the components with respect to the additional degrees of freedom have non-zero values when subjected to a distributed load. These values do not have a physical but just a numerical meaning. 


\subsection{Equilibrium equation in nonlinear analysis}

The governing equations in $\$ 2.1$ provide only an equilibrium state of the rates of internal and external forces at one moment and do not represent an equality of internal and external forces. The discretized relation, which must be satisfied in the equilibrium state, is

$$
\{\underline{\mathbf{Q}}\}=\{\underline{\dot{\mathbf{F}}}\}
$$

In the case of the quasi-static state in which the inertia force can be neglected, the following equations can be obtained by integrating both sides of Eq. (23) from the initial time 0 to the current time $t$ :

$$
\begin{aligned}
& \{\underline{\mathbf{Q}}\}=\{\underline{\mathbf{F}}\} \\
& \{\underline{\mathbf{Q}}\} \equiv \int_{0}^{t}\{\underline{\mathbf{Q}}\} \mathrm{d} t \\
& \{\underline{\mathbf{F}}\} \equiv \int_{0}^{t}\{\underline{\mathbf{F}}\} \mathrm{d} t
\end{aligned}
$$

The nodal displacement vector is also given in the same manner.

$$
\{\underline{\mathbf{U}}\} \equiv \int_{0}^{t}\{\underline{\dot{\mathbf{U}}}\} \mathrm{d} t
$$

Equation (24) is nonlinear with respect to displacement, because the relationship between displacement and internal force is generally nonlinear. For this reason, convergent calculation by a nonlinear implicit method should be introduced to solve the equation. In the present formulation, the tangent stiffness matrix $[\underline{\mathbf{K}}]$ is not always consistent with the internal force; therefore, the modified Newton method is used, and a converged solution can be obtained in nonlinear analysis.

\section{Numerical examples}

In this section, four numerical examples are presented. The first example is a linear deformation problem and the others take into account geometrical nonlinearity. In each analysis, the phenomenon in which a stiffer solution is obtained (called locking) may occur when conventional finite elements are used. The convergence of the solution with respect to a number of elements and the robustness against element distortion are investigated. The results obtained by the conventional 4-, 8-, and 9-node isoparametric finite elements (QD4, QD8, and QD9) are also shown for comparison. Full Gaussian integration is adopted for all types of elements; i.e., the numbers of integration points are $2 \times 2$ for QD4 and $3 \times 3$ for QD8, QD9, and the present element. The features of the finite elements used in this study are summarized in Table 1. It is assumed that the material has isotropic elasticity with Young's modulus $E$ and Poisson's ratio $v$. In all nonlinear analysis, the number of increments is determined as it provides a sufficiently converged solution.

\subsection{Linear bending of a cantilever beam}

First, a linear bending analysis of a cantilever beam subjected to lumped loading at the tip is conducted to evaluate the performance of the present element in a linear problem. The ratio between the length and thickness is $L / t=100$. The number of elements in the thickness direction is always one and that in the longitudinal direction varies. To investigate the robustness against element distortion, the analysis mesh is distorted, as shown in Fig. 2. $h$ is the characteristic length of the element, and parameter $\beta$ characterizes element distortion, which takes the values of $0 \leq \beta \leq 1 . \beta=0$ corresponds to a regular rectangle mesh and the element degenerates into a triangular shape when $\beta=1$. Note that the present 
Table 1 Features of elements used in the present study.

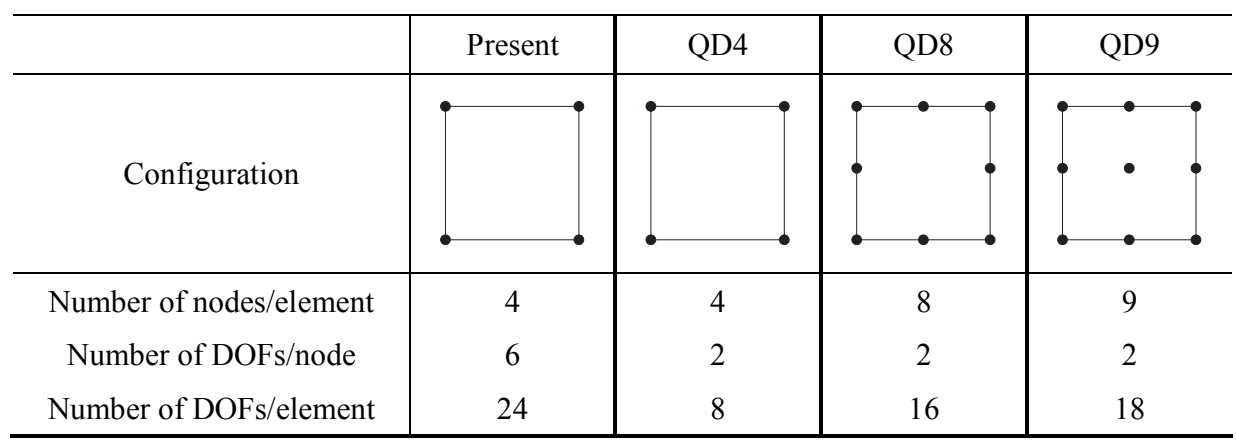

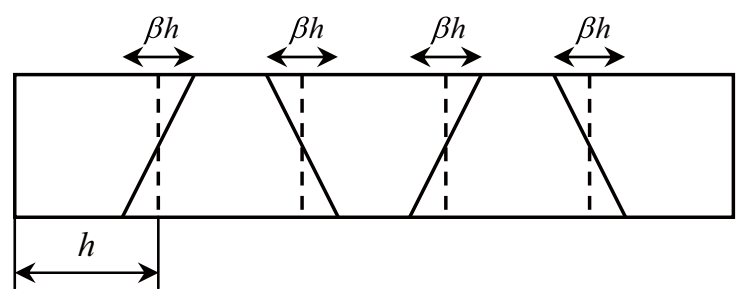

Fig. 2 Definition of element distortion.

element can be used as a triangular element when $\beta=1$.

The theoretical tip deflection is calculated as

$$
u=\frac{1}{3} \frac{P L^{3}}{E I}+\frac{1}{10} \frac{P L t^{2}}{G I}
$$

Here $G$ is the shear modulus, and the geometric moment of inertia $I$, with a square cross area $A$ is expressed as $I=A t^{2} / 12$. The first and second terms on the right-hand side of Eq. (28) are caused by bending and shear deformation, respectively. In the present condition, the bending deformation is dominant and the contribution of shear deformation is only $0.006 \%$, and thus, it can be considered negligible.

The analysis results when the mesh has no distortion $(\beta=0.00)$ are indicated in Table 2. The following results are normalized by the theoretical solution obtained by Eq. (28). In this table, it is noted that the numbers of degrees of freedom are different even if the numbers of elements are identical. In the present analysis, the degrees of freedom of the present element, QD8 and QD9 are almost three times as that of QD4, e.g., in the case of the 100 elements mesh, 12,012, 4,004, 11,006 and 12,006 for the present, QD4, QD8 and QD9, respectively. The convergence with respect to the number of degrees of freedom is shown in Fig. 3. QD8 and QD9 show nearly same convergence because the elements have no distortion. The present element gives almost exact solution even if the number of elements is 10 , and the error is always less than the quadratic elements. The convergence rate is mostly the same as that of the quadratic elements while QD4 shows considerably slow convergence because of shear locking. Next, the number of elements is fixed as 100 and mesh distortion is imposed. Each element becomes a regular tetragon when $\beta=0.00$, because $L / t=100$. The effect of $\beta$ on the analysis error is shown in Table 3 . The accuracy of the present element and QD9 are unaffected by element distortion, even if the element degenerates into a triangular shape with $\beta=1.00$. On the other hand, the accuracy of QD4 and QD8 are reduced due to element distortion. Moreover, the convergence with respect to the number of degrees of freedom is illustrated in Fig. 4. The convergence ratio is better than that of conventional elements as well as those with a regular mesh, and the present element has high robustness against mesh distortion. In Figs. 3 and 4, the present element 
Table 2 Bending of thin cantilever beam: normalized tip deflection versus to number of elements.

\begin{tabular}{c|ccc}
\hline & \multicolumn{3}{|c}{ Number of elements } \\
& 10 & 100 & 1000 \\
\hline Present & 1.000 & 1.000 & 1.000 \\
QD4 & 0.020 & 0.667 & 0.995 \\
QD8 & 0.998 & 1.000 & 1.000 \\
QD9 & 0.998 & 1.000 & 1.000 \\
\hline
\end{tabular}

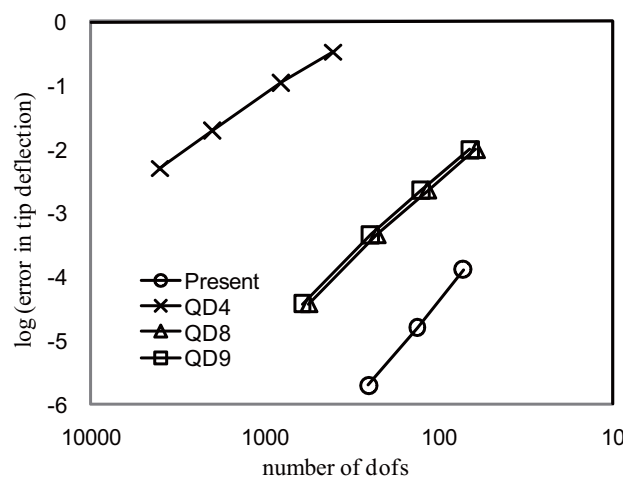

Fig. 3 Bending of a thin cantilever beam with a regular mesh: error vs. number of degrees of freedom.
Table 3 Bending of thin cantilever beam: normalized tip deflection respect to element distortion.

\begin{tabular}{c|ccc}
\hline & \multicolumn{3}{|c}{ Element distortion $\beta$} \\
& 0.00 & 0.50 & 1.00 \\
\hline Present & 1.000 & 1.000 & 1.000 \\
QD4 & 0.667 & 0.460 & 0.238 \\
QD8 & 1.000 & 0.991 & 0.871 \\
QD9 & 1.000 & 1.000 & 1.000 \\
\hline
\end{tabular}

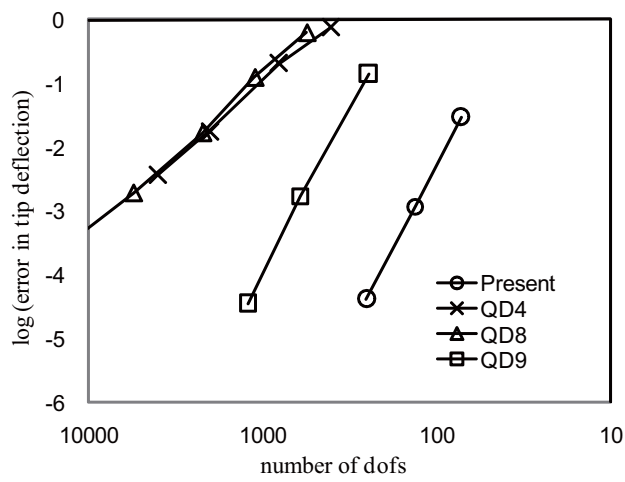

Fig. 4 Bending of a thin cantilever beam with a distorted mesh ( $\beta=0.99$ ): error vs. number of degrees of freedom.

always shows better performance than QD4, QD8 and QD9 for the same number of degrees of freedom.

These results confirm that the proposed element shows performance equal to or better than QD8 and QD9 for linear bending problems, and no shear locking occurs, although it obviously arises with QD4, whose geometrical shape is the same as the present one.

\subsection{Buckling of toggle structure}

The second example is the snap-through buckling analysis of the toggle structure shown in Fig. 5(a). The displacements are constrained at both ends, and the displacement history with respect to loading at the top of structure is shown in Fig. 5(b). Only one half of the structure is analyzed because of its symmetry. The loading control with the arc-length method is conducted and the buckling load, or the local maximum loading, is evaluated. The convergence solution obtained with a sufficiently fine mesh is $P_{c r}=4.964 \times 10^{-5} \mathrm{EA}$ with Young's modulus $E$ and a square cross section whose area is $A$. The number of elements in the thickness direction is always one and that in the length direction varies.

Figure 6 shows convergence versus the number of elements when the mesh has no distortion. The results are normalized by the convergence solution. For 10 elements, QD8, QD9, and the present element show a slightly higher buckling load, and in the results for 100 or more elements, all three types of elements yield almost the exact same results. On the other hand, QD4 generally presents a stiffer response, and higher buckling load is estimated due to shear locking, as in the previous example. Next, the number of elements in the longitudinal direction is fixed as 100 and the elements are distorted as in \$3.1. Figure 7 illustrates the relationship between the element distortion and the estimated buckling load. For the present element, there is no reduction in the accuracy even if the mesh is strongly distorted. Figures 8 and 9 show the analysis error with respect to the number of degrees of freedom using nondistorted $(\beta=0.00)$ and distorted $(\beta=0.99)$ meshes. The present 


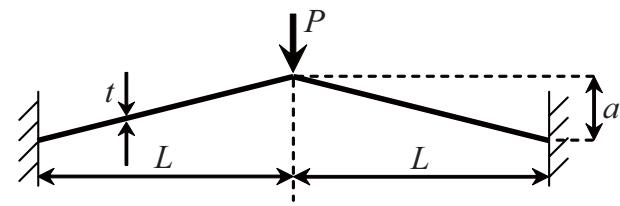

$L / t=100$

$L / a=10$

(a) Analysis model.

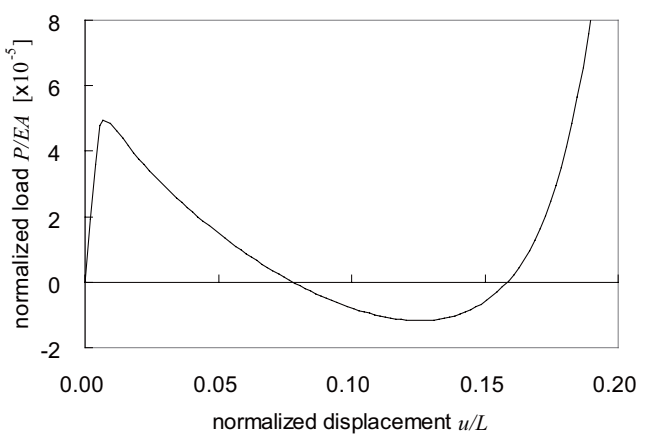

(b) Loading history.

Fig. 5 Snap-through buckling of a toggle structure.

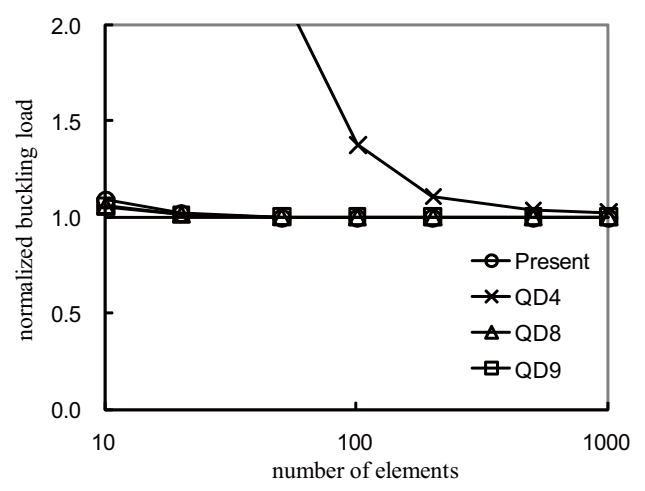

Fig. 6 Buckling of a toggle: normalized buckling load vs. number of elements.

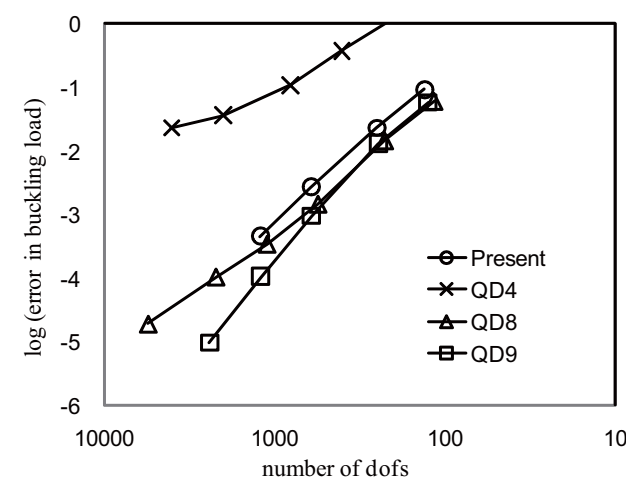

Fig. 8 Buckling of a toggle with a regular mesh: error vs. number of number of degrees of freedom.

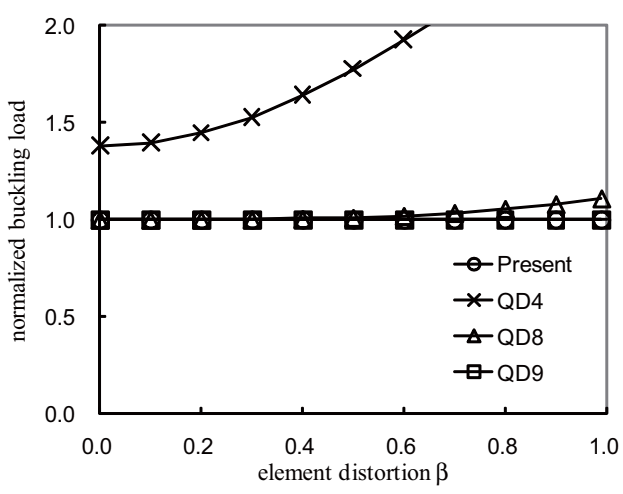

Fig. 7 Buckling of a toggle: normalized buckling load vs. element distortion.

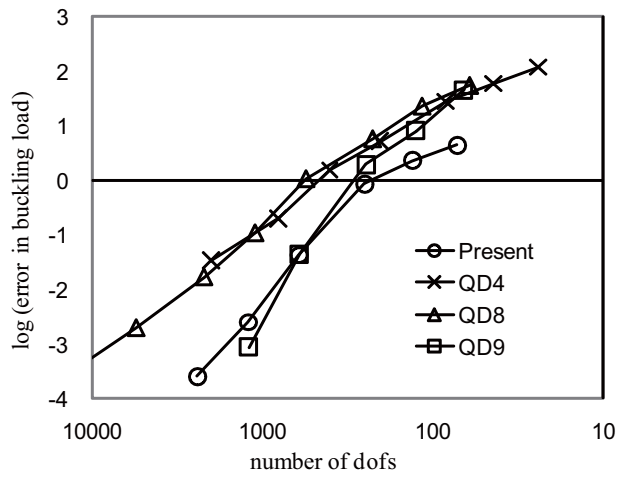

Fig. 9 Buckling of a toggle with a distorted mesh $(\beta=0.99)$ : error vs. number of number of degrees of freedom.

element shows tendencies similar to QD8 and QD9, and it can be said that these elements demonstrate robust convergence against mesh distortion.

\subsection{Rolling up of plate}

The third problem, a rolling up analysis of a thin plate, is shown in Fig. 10. In the present case, the Poisson's ratio $v$ is set as zero. The cross-sectional shape of the beam is a rectangle with area $A$, and the specimen is subjected to moment $M$. Under these conditions, the rotation angle at the tip $\phi$ can theoretically be given as

$$
\phi=12 M L / E A t^{2}
$$




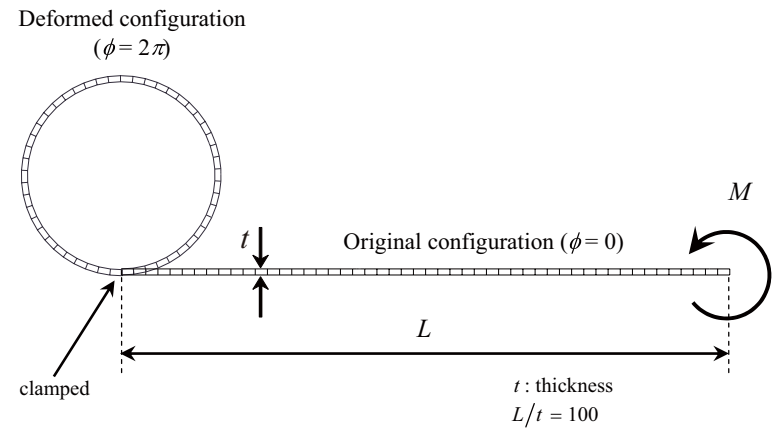

Fig. 10 Rolling-up of a plate.

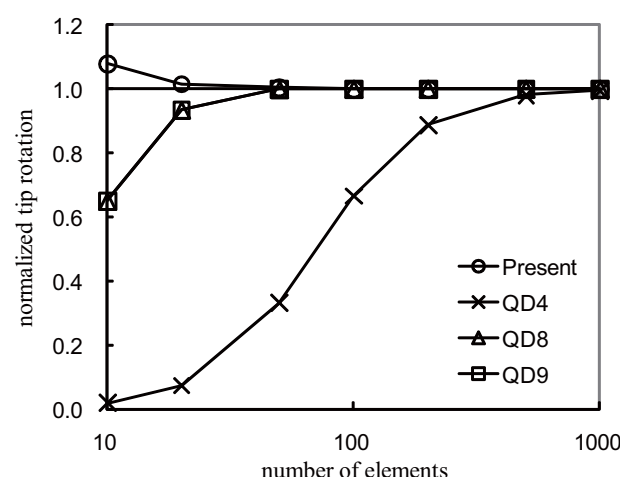

Fig. 11 Rolling up of a plate: normalized tip rotation vs. number of elements.

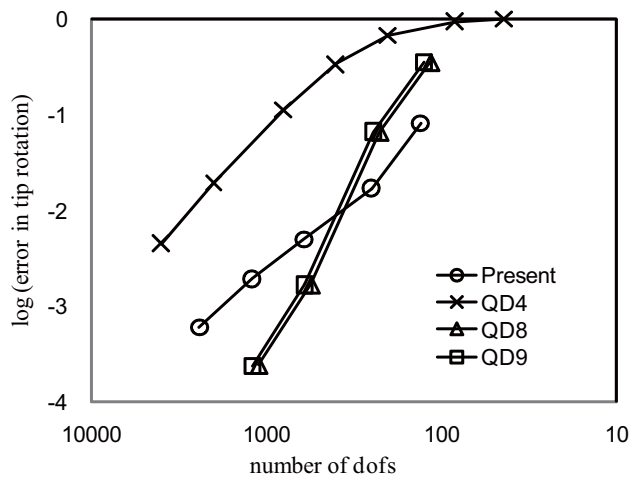

Fig. 13 Rolling-up of a plate with a regular mesh: error vs. number of number of degrees of freedom.

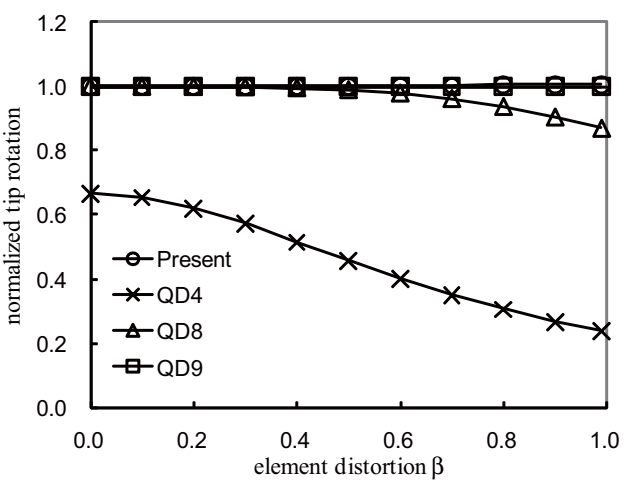

Fig. 12 Rolling up of a plate: normalized tip rotation vs. element distortion.

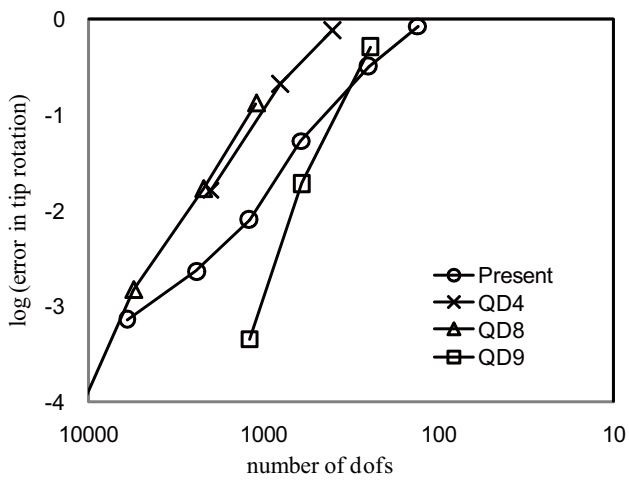

Fig. 14 Rolling-up of a plate with a distorted mesh ( $\beta=0.99$ ): error vs. number of number of degrees of freedom.

In this analysis, the plate tip is subject to moment $M=\pi E A t^{2} / 6 L$, and the exact solution is $\phi=2 \pi$. The number of elements in the thickness direction is always one and that in the length direction varies. Element distortion takes place in the same manner as in $\S \S 3.1$ and 3.2 .

The convergence in tip rotation with respect to the number of elements is shown in Fig. 11 ; in this case, the element has no distortion or $\beta=0.00$. All results are normalized by the theoretical value using Eq. (29). The proposed element gives only $8.1 \%$ error in tip rotation even for 10 elements. On the other hand, the conventional elements provide a stiffer solution, especially with a smaller number of elements, even if QD8 or QD9 are used. For second-order elements QD8 and QD9, this is caused by membrane locking, which results from unphysical membrane strain in curved elements. In the case of QD4, shear locking 


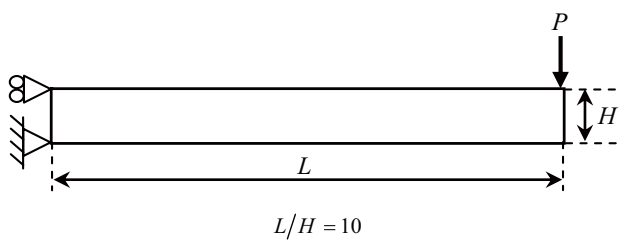

Fig. 15 Bending of a thick beam.

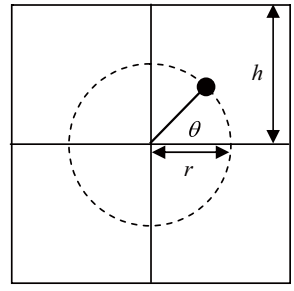

(a) Definition of distortion

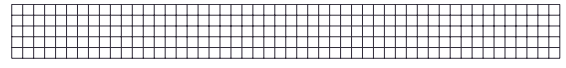

(b) Regular mesh.

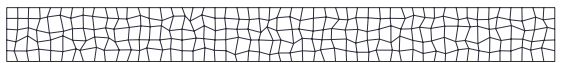

(c) Distorted mesh with $\gamma=0.80$.

Fig. 16 Element distortion in Section 3.4.

with unphysical strain in pure bending degrades the convergence. Figure 12 shows the results obtained using distorted meshes. The number of elements is fixed to 100 , which gives a regular tetragon element. The present element provides an almost exact solution even if $\beta=0.99$ and the robustness against element distortion is similar to that of QD9. Figures 13 and 14 indicate errors in the tip rotation with respect to the number of degrees of freedom for regular and distorted meshes $(\beta=0.99)$. With a regular mesh, the present element provides results as good as, or better than, those of QD8 or QD9. For a distorted mesh, the proposed element shows good performance, especially with a small number of degrees of freedom, although the rate of convergence is slightly slower than that for QD9.

\subsection{Bending of quasi-incompressible and thick beams}

As the last example, bending of quasi-incompressible and thick beams shown in Fig. 15 is analyzed. To reproduce the incompressible condition, Poisson's ratio $v$ is set as 0.499 and a plane strain state is assumed. Under this condition, volumetric locking may occur in numerical analysis. It is subjected to lumped loading $P=5 \times 10^{-4} \mathrm{EA}$ at the beam tip, and the corresponding tip deflection is about $0.1 L$. Therefore, geometrical nonlinearity cannot be neglected. In this problem, the converged solution with an extraordinary fine mesh is used as a reference because no theoretical solution is available. The results are normalized using this reference.

In this analysis, the element distortion is defined as the following way. Every element in the original mesh is square shaped, and each node is randomly moved as illustrated in Fig. 16(a). Here $h$ is the characteristic length of the original mesh. $\theta$ and $r$ are random numbers in ranges of $0 \leq \theta<2 \pi$ and $0 \leq r<\gamma h / 2$, respectively, with the parameter $\gamma$, which characterizes element distortion. The generated mesh is regular when $\gamma=0$, and the mesh distortion becomes stronger with increasing $\gamma$. The examples of regular and distorted meshes are illustrated in Figs. 16(b) and (c).

The convergence of tip deflection versus the number of elements in the length direction is shown in Fig. 17. The element has no distortion, i.e., $\gamma=0.00$, and the ratio of the length to the height of the element is always unity, indicating each element is always a regular tetragon. The results with the present element, QD8, and QD9 are almost the same, even if the number of elements in the thickness direction is one (a total of five elements). On the other hand, QD4 yields a very stiff response because of volumetric locking, which is caused by the constraint of (almost) incompressible deformation. These results indicate that volumetric locking does not occur with the proposed element, although it cannot be avoided 


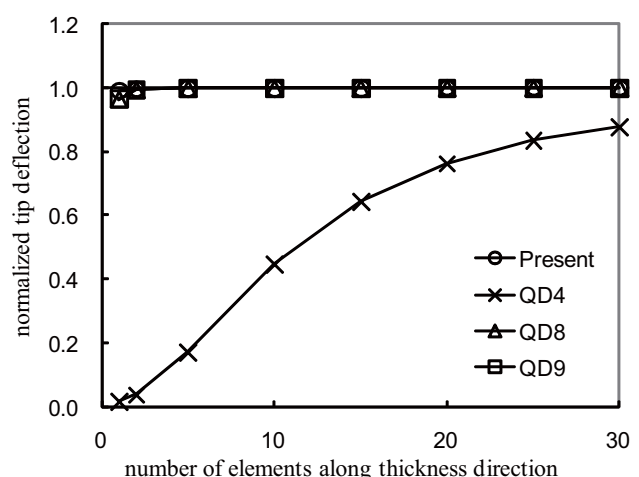

Fig. 17 Bending of a thick beam: normalized tip deflection vs. number of elements.

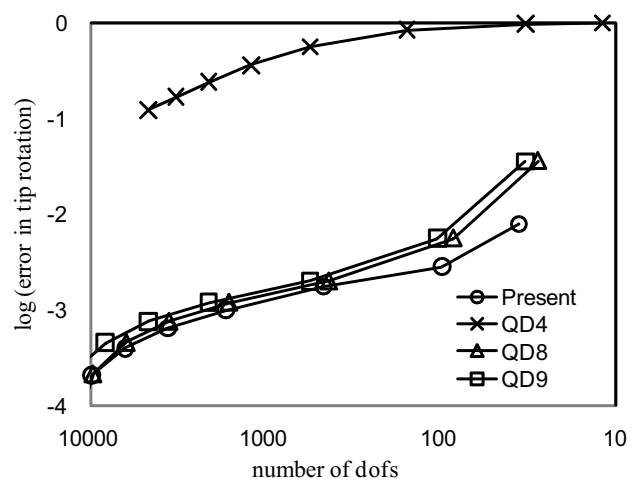

Fig. 19 Bending of a thick beam with a regular mesh: error vs. number of degrees of freedom.

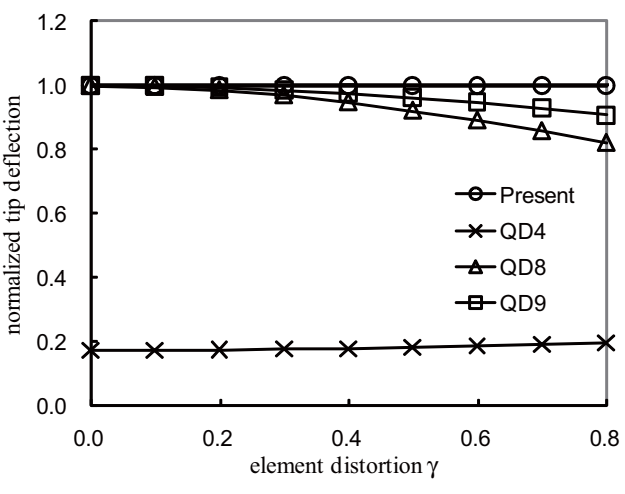

Fig. 18 Bending of a thick beam: normalized tip deflection vs. element distortion.

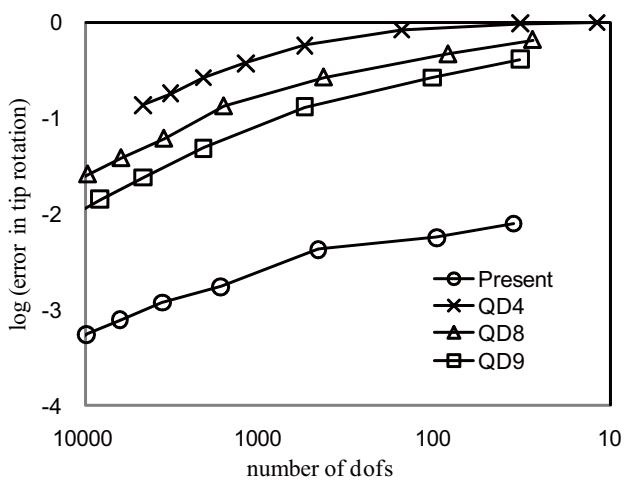

Fig. 20 Bending of a thick beam with a distorted mesh $(\gamma=0.80)$ : error vs. number of degrees of freedom.

with QD4. Figure 18 shows the results with distorted meshes. The number of elements is fixed as $50 \times 5$. The solution obtained by the present element is almost exactly equal to that of the reference, even if $\gamma=0.80$, although element distortion affects the precision of the solution with all conventional elements, even for QD9. Figures 19 and 20 show errors in tip deflection with respect to the number of degrees of freedom obtained using regular and distorted meshes with $\gamma=0.80$. In the results with a distorted mesh, the convergence ratio of the presented element shows very high performance relative to QD8 and QD9, although with the regular mesh, the rate is about the same.

\section{Concluding remarks}

In this paper, a novel in-plane finite element is presented in the framework of the generalized finite element method based on the partition of unity method, and it is introduced into geometrically nonlinear analyses. Motivated by Wilson's nonconforming element, which is a well-known way to improve the accuracy of finite element, the 4-node conventional linear element is enriched by the reduced quadratic polynomial terms, and it can reproduce the quadratic deformation mode with only corner nodes. The formulation is based on the rate form of the virtual work principle and is obtained by a simple extension of the standard FEM. Two features of the present element should be emphasized. First, it is a compatible element, although the original Wilson's formulation is an incompatible one. Second, the present element can avoid linear dependency, which is a well-known problem of generalized finite elements. In addition, the constitutive relation can be arbitrary as long as it takes the general rate form of Eq. (3), although only isotropic elasticity is considered in this paper. 
Four typical numerical examples, one linear problem and three geometrically nonlinear analyses are presented. The convergence of the analysis solution and its robustness for element distortion are investigated and the results are compared with those of the standard displacement-based first- and second-order finite elements. The proposed element provides good solution convergence, which is as good as or better than that of the conventional second-order elements, QD8 and QD9. It is shown that a high-precision solution is given when the mesh is strongly distorted, and no shear, membrane, or volumetric locking arises. In particular, it is noted that it avoids membrane and volumetric locking. The former issue occurs even if conventional quadrilateral elements are used. Volumetric locking often arises when an incompressible material, such as a hyperelastic or elastoplastic model, is assumed.

All these results suggest that the proposed approach provides good performance for analysis convergence and high robustness against element distortion in geometrically nonlinear problems. Therefore, it can be said that the presented element is applicable to many practical examples involving geometrical nonlinearity and is compatible with the conventional finite element framework.

\section{References}

1. Babuška, I. and Osborn, J.E., Generalized Finite Element Methods: Their Performance and Their Relation to Mixed Methods, SIAM Journal on Numerical Analysis, Vol. 20 (1983), pp. 510-36.

2. Zienkiewicz, O.C., Generalized Finite Element Method: State of the Art and Future Directions, Transactions of the ASME Journal of Applied Mechanics, Vol. 50 (1983), pp. 1210-1217.

3. Babuška, I., Caloz, G. and Osborn, J., Special Finite Element Methods for a Class of Second Order Elliptic Problems with Rough Coefficients, SIAM Journal on Numerical Analysis, Vol. 31 (1994), pp. 945-981.

4. Melenk, J.M. and Babuška, I., The Partition of Unity Finite Element Method: Theory and Application, Computer Methods in Applied Mechanics and Engineering, Vol. 139 (1996), pp. 289-314.

5. Babuška, I. and Melenk, J.M., The partition of unity finite element method, International Journal for Numerical Methods in Engineering, Vol. 40 (1997), pp. 727-758.

6. Taylor, R.L., Zienkiewicz, O.C. and Oñate, E., A Hierarchical Finite Element Method Based on the Partition of Unity, Computer Methods in Applied Mechanics and Engineering, Vol. 152 (1998), pp. 73-84.

7. Babuška, I., Banerjee, U. and Osborn, E., On Principles for the Selection of Shape Functions for the Generalized Finite Element Method, Computer Methods in Applied Mechanics and Engineering, Vol. 191 (2002), pp. 5595-5629.

8. Babuška, I., Banerjee, U. and Osborn, J., Generalized Finite Element Methods-Main Ideas, Results and Perspective, International Journal of Computational Methods, Vol. 1 (2004), pp. 67-103.

9. Babuška, I., Banerjee, U. and Osborn, J., Survey of Meshless and Generalized Finite Element Methods, Acta Numerica, Vol. 12 (2003), pp. 1-125.

10. Strouboulis, T., Copps, K. and Babuška, I., The Generalized Finite Element Method, Computer Methods in Applied Mechanics and Engineering, Vol. 190 (2001), pp. 4081-4193.

11. Allman, D.J., A Compatible Triangular Element Including Vertex Rotations for Plane Elasticity Analysis, Computers \& Structures, Vol. 19 (1984), pp. 1-8.

12. Allman, D.J., A Quadrilateral Finite Element Including Vertex Rotations for Plane Elasticity Analysis, International Journal for Numerical Methods in Engineering, Vol. 26 (1988), pp. 717-730.

13. Hughes, T.J.R., On Drilling Degrees of Freedom, Computer Methods in Applied 
Mechanics and Engineering, Vol. 72 (1989), pp. 105-121.

14. Sekiguchi, M. and Kikuchi, N., Re-Examination of Membrane Elements with Drilling Freedom, Proceedings of Fifth World Congress on Computational Mechanics, (2002).

15. Barros, F.B., Proenca, S.P.B. and de Barcellos, C.S., Generalized Finite Element Method in Dtructural Nonlinear Analysis-A p-Adaptive Strategy, Computational Mechanics, Vol. 33 (2004), pp. 95-107.

16. Terada, K., Asai, M. and Yamagishi, M., Finite Cover Method for Linear and Nonlinear Analyses of Heterogeneous Solids, International Journal for Numerical Methods in Engineering, Vol. 58 (2003), pp. 1321-1346.

17. Taylor, R.L., Beresford, P.J. and Wilson, E.L., A Non-Conforming Element for Stress Analysis, International Journal for Numerical Methods in Engineering, Vol. 10 (1976), pp. 1211-1219.

18. Zienkiewicz, O.C. and Taylor, R.L., The Finite Element Method: Fifth Edition, Vol. 1, (2000), Butterworth Heinemann. 